\title{
A Local Hubble Bubble from Type la Supernovae?
}

\section{Citation}

Zehavi, Idit, Adam G. Riess, Robert P. Kirshner, and Avishai Dekel. 1998. "A Local Hubble Bubble from Type la Supernovae?" The Astrophysical Journal 503 (2): 483-91. https:// doi.org/10.1086/306015.

\section{Permanent link}

http://nrs.harvard.edu/urn-3:HUL.InstRepos:41399824

\section{Terms of Use}

This article was downloaded from Harvard University's DASH repository, and is made available under the terms and conditions applicable to Other Posted Material, as set forth at http:// nrs.harvard.edu/urn-3:HUL.InstRepos:dash.current.terms-of-use\#LAA

\section{Share Your Story}

The Harvard community has made this article openly available.

Please share how this access benefits you. Submit a story.

\section{Accessibility}


The Astrophysical Journal, 503:483-491, 1998 August 20

(C) 1998. The American Astronomical Society. All rights reserved. Printed in U.S.A.

\title{
A LOCAL HUBBLE BUBBLE FROM TYPE Ia SUPERNOVAE?
}

\author{
Idit Zehavi, ${ }^{1}$ AdAm G. Riess, ${ }^{2}$ Robert P. KiRShNER, ${ }^{3}$ AND Avishai DeKel ${ }^{1,4}$ \\ Received 1997 September 16; accepted 1998 April 1
}

\begin{abstract}
We analyze the monopole in the peculiar velocities of 44 Type Ia supernovae (SNe Ia) to test for a local void. The sample extends from 20 to $300 h^{-1} \mathrm{Mpc}$, with distances, deduced from light-curve shapes, accurate to $\sim 6 \%$. Assuming $\Omega_{m}=1$ and $\Omega_{\Lambda}=0$, the most significant deviation we find from the Hubble law is an outward flow of $6.5 \% \pm 2.2 \%$ inside a sphere of radius $70 h^{-1} \mathrm{Mpc}$, as would be produced by a void of $\sim 20 \%$ underdensity surrounded by a dense shell. This shell roughly coincides with the local great walls. Monte Carlo analyses, using Gaussian errors or bootstrap resampling, show the probability for chance occurrence of this result out of a pure Hubble flow to be $\sim 2 \%$. The monopole could be contaminated by higher moments of the velocity field, especially a quadrupole, which are not properly probed by the current limited sky coverage. The void would be less significant if $\Omega_{m}$ is low and $\Omega_{\Lambda}$ is high. It would be more significant if one outlier is removed from the sample, or if the size of the void is constrained a priori. This putative void is not in significant conflict with any of the standard cosmological scenarios. It suggests that the Hubble constant as determined within $70 h^{-1} \mathrm{Mpc}$ could be overestimated by $\sim 6 \%$, and the local value of $\Omega$ may be underestimated by $\sim 20 \%$. While the present evidence for a local void is marginal in this data set, the analysis shows that the accumulation of SN Ia distances will soon provide useful constraints on elusive and important aspects of regional cosmic dynamics.
\end{abstract}

Subject headings: cosmology: observations - cosmology: theory — galaxies: distances and redshifts large-scale structure of universe - supernovae: general

\section{INTRODUCTION}

Large-scale redshift surveys of galaxies show underdense regions of typical extent $\sim 50 h^{-1} \mathrm{Mpc}$. These "voids" appear to be bordered by dense "walls" (Kirshner et al. 1981; Huchra et al. 1983 [CfA]; Broadhurst et al. 1990; Shectman et al. 1996 [Las Campanas Redshift Survey (LCRS)]). In particular, maps of our cosmological neighborhood display the Great Wall of Coma and the Southern Wall, which appear to connect into a shell-like structure of radius $70-80 h^{-1} \mathrm{Mpc}$ about the Local Group (Geller \& Huchra 1989 [CfA2]; da Costa et al. 1994 [Southern Sky Redshift Survey 2 (SSRS2)]). The volume encompassed by this structure appears to be of lower density.

Despite these impressive maps, it is difficult to quantify the large-scale radial density profile of this region. First, the true galaxy density is hard to distinguish from the sample selection function when the structure of interest approaches the sample size. Second, we do not know how well galaxies trace mass. And third, portions of the galaxy distribution are obscured from our viewpoint within the Milky Way.

The imprint of wall and void structure on the power spectrum may possibly be associated with excess power observed at a wavelength of $\sim 100-150 h^{-1} \mathrm{Mpc}$ (e.g., Broadhurst et al. 1990; Landy et al. 1996; Einasto et al. 1997). This scale might be naturally attributed to the scale of the cosmological horizon after the universe became matter dominated and before the plasma recombined, though this peak is only expected to be significant for rela-

\footnotetext{
${ }^{1}$ Racah Institute of Physics, Hebrew University, Jerusalem 91904, Israel.

${ }^{2}$ Astronomy Department, University of California, Berkeley, CA 94720

${ }^{3}$ Harvard-Smithsonian Center for Astrophysics, 60 Garden Street, Cambridge, MA 02138

${ }^{4}$ UCO/Lick Observatory, University of California, Santa Cruz, CA 95064
}

tively high values of the baryon density (Hu \& White 1996).

A local void has been proposed as one way to reconcile the age of the universe based on the Hubble expansion with the ages of globular clusters within the framework of the Einstein-de Sitter cosmology (e.g., Turner, Cen, \& Ostriker 1992; Bartlett et al. 1995) Measurements of the Hubble constant within the void would overestimate the universal value by $\delta \rho / \rho \approx-3 \delta H / H$. Indeed, the values obtained for the Hubble constant from the longest-range distance indicators, the SNe Ia (Jacoby et al. 1992; Sandage \& Tammann 1993; Tammann \& Sandage 1995; Hamuy et al. 1995, 1996b; Riess, Press, \& Kirshner 1995a, 1996; Branch, Nugent, \& Fisher 1997) and the gravitational lenses (Falco et al. 1997; Keeton \& Kochanek 1997) are typically smaller than values obtained more locally using Tully-Fisher (TF) distance indicators (Kennicutt, Freedman, \& Mould 1995; Mould et al. 1995; Freedman et al. 1994; Freedman 1997, Giovanelli et al. 1997). A local void would also imply that local estimates of $\Omega$ underestimate the global value of $\Omega$. Finally, a local outflow would reduce the distances derived from TF peculiar velocities for features such as the Great Attractor, bringing them into better agreement with the positions derived from redshift surveys (Sigad et al. 1998).

It is important to separate impressions and theoretical wishes from firmly established observational facts. Attempts to establish monopole deviations from pure Hubble flow have not yet produced conclusive results. For example, Shi (1997) claimed finding a monopole gradient in subsamples of the Mark III catalog of Tully-Fisher peculiar velocities (Willick et al. 1997a). But he also found a marginal monopole gradient of the opposite sign in the peculiar motions of rich clusters based on their brightest members as standard candles (Lauer \& Postman 1992, 1994). Finally, he found no significant deviation in an early subsample of 20 supernovae (Riess et al. 1996). None of these data sets was ideal for testing for a void of radius $\sim 70 h^{-1} \mathrm{Mpc}$ : the Mark III data include only a small number of galaxies beyond $70 h^{-1}$ 
Mpc, the earlier supernova sample is too sparse, and the rich clusters, while of comparable abundance to the current supernova sample within $70 h^{-1} \mathrm{Mpc}$, have a much larger error per cluster. Kim et al. (1997), based on comparisons of supernova distances at high redshifts $(z \sim 0.4)$ and low redshifts $(z<0.1)$, set a $2 \sigma$ upper limit of $10 \%$ for an outflow within the local $300 h^{-1} \mathrm{Mpc}$, but their sample has only a few supernovae within $70 h^{-1} \mathrm{Mpc}$, which is the suggested domain of the putative void.

We now have a sample of $44 \mathrm{SNe}$ Ia from the Calan/ Tololo Survey (Hamuy et al. 1993a, 1996a) and from the CfA supernova program (Riess 1996; Riess et al. 1998), which reaches the threshold for an interesting assessment of the dynamical signature of a void contained within the Great Walls. The nearest supernovae in this sample are at distances of $\sim 20 h^{-1} \mathrm{Mpc}$, the farthest are beyond $300 \mathrm{~h}^{-1}$ $\mathrm{Mpc}$, the median is at $\simeq 95 h^{-1} \mathrm{Mpc}$, and 17 supernovae lie inside $70 h^{-1} \mathrm{Mpc}$. This distribution in distance is well suited for detecting a monopole perturbation within 50-100 $h^{-1} \mathrm{Mpc}$ while determining the universal Hubble flow outside this sphere. These are the scales which have been suggested and where the standard cosmological theories predict perturbations on the order of several percent (e.g., Wang, Spergel, \& Turner 1998). Because supernova light curves provide distances with a precision of $5 \%-8 \%$, we believe it is worth carrying out a preliminary analysis with only 44 tracers. This sample has already been used to put constraints on the global Hubble flow and the dipole motion (Hamuy et al. 1995, 1996b; Riess, Press, \& Kirshner 1995a, 1995b, 1996), and the nearby sample has been used to assess how well the velocities of supernovae match the gravity field inferred from galaxy redshift surveys (Riess et al. 1997).

In $\S 2$ we describe the data. In $\S 3$ we measure the peculiar monopole and evaluate its significance in an Einstein-de Sitter universe. In $\S 4$ we explore the effect of different cosmologies, error estimates and higher velocity-field moments on our results. In $\S 5$ we discuss the implications of our results.

\section{DATA}

Our data consist of $44 \mathrm{SNe}$ Ia light curves with distances (d) inferred by the method of multicolor light-curve shapes (MLCSs) (Riess, Press, \& Kirshner 1996, hereafter RPK) and redshifts ( $z$ ) (Hamuy et al. 1996a; Riess 1996; Riess et al. 1998). The MLCS method can measure distances with $\sim 6 \%$ precision by accounting for variations in SN Ia luminosity and correcting for line-of-sight extinction.

The inferred distance is a "luminosity" distance. To account for cosmological curvature effects, we define the peculiar velocity as $u=d_{l}(z)-d$, where $d_{l}(z)$ is the luminosity distance (in units of kilometers per second) corresponding to redshift $z$ for the assumed values of the cosmological parameters $\Omega_{m}$ and $\Omega_{\Lambda}$. It is given by

$$
\begin{aligned}
d_{l}(z) & =\frac{c(1+z)}{\left|\Omega_{k}\right|^{1 / 2}} S_{k}\left\{\left|\Omega_{k}\right|^{1 / 2}\right. \\
& \left.\times \int_{0}^{z} d z^{\prime}\left[\left(1+z^{\prime}\right)^{2}\left(1+\Omega_{m} z^{\prime}\right)-z^{\prime}\left(2+z^{\prime}\right) \Omega_{\Lambda}\right]^{-1 / 2}\right\},
\end{aligned}
$$

where $k$ is the curvature parameter, $\Omega_{k}=1-\Omega_{m}-\Omega_{\Lambda}$, $S_{0}(x) \equiv x, S_{+1} \equiv \sin$, and $S_{-1} \equiv \sinh$. Every $z$ hereafter actually refers to $d_{l}(z)$.
"Formal" estimates of the distance errors for the individual supernovae, including the intrinsic scatter in the distance indicator and the measurement errors, are provided by the covariance matrix of the MLCS fit (RPK), $\Delta_{\text {MLCS }}$. These internal errors are typically $5 \%-8 \%$ of the distance. Additional systematic errors in the distances are uncertain, so we adopt a conservative approach by adding in quadrature $\Delta_{\text {add }}=0.05$ mag in our "standard" study case (in $\S 3$ ), and later discuss the implications of varying $\Delta_{\text {add }}$ in the range $0-0.1 \mathrm{mag}$.

\section{PECULIAR MONOPOLE}

\subsection{Fitting a Monopole Profile}

Figure 1 shows the radial peculiar velocities of the 44 supernovae, $u$, translated to the corresponding relative deviations from a pure Hubble flow, $\delta_{H} \equiv \delta H / H=u / d$, at the inferred distances $d$, assuming (in this section) $\Omega=1$ and $\Lambda=0$. The plot suggests positive $\delta_{H}$, an outflow, inside 70 $h^{-1} \mathrm{Mpc}$, and possibly a marginal indication for an inflow in the surrounding shell 70-110 $h^{-1} \mathrm{Mpc}$, before the flow converges to a global Hubble expansion at larger distances.

The 26th supernova from the Local Group at $d \simeq 107$ $h^{-1} \mathrm{Mpc}$, named SN 1992bl, is the most discrepant point in this plot. Its inferred peculiar velocity of $\sim 2400 \mathrm{~km} \mathrm{~s}^{-1}$ is a $\sim 4 \sigma$ deviation from the global Hubble flow. As described below, the exclusion of this outlier from the analysis makes a difference in the significance of the results. Though we have no reason to think that SN 1992bl is incorrectly analyzed, and we have not found anything obvious that makes it an a priori suspect, we want to evaluate the impact of this single point on the significance of the void, and we therefore report the results both with and without this outlier.

While visual inspection of Figure 1 suggests the possibility of a local void, our approach is to use a statistical analysis to provide a quantitative estimate of the significance of the void. The Hubble constant $H$ within a given volume is determined by minimizing the error-weighted

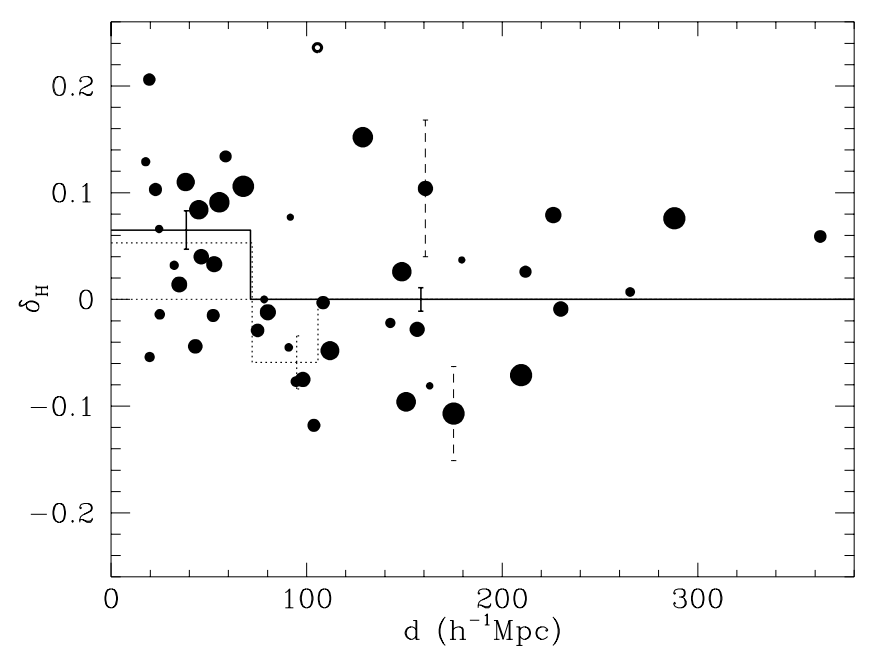

Fig. 1.-Monopole profile: $\delta_{H}$ vs. inferred distance $d$. The individual supernovae are marked as circles of radii inversely proportional to the errors (the actual errors are shown for two representative supernovae to calibrate the size of the symbols). Overlaid are the best-fit two-zone (solid line) and three-zone (dashed line) monopole models, together with the corresponding errors of the fits. The global $H$ is determined from the outer region. The outlier SN 1992bl is marked by the white dot. 
sum of residuals over the supernovae,

$$
\chi^{2}=\sum_{i}\left[\log \left(z_{i} / d_{i}\right)-\log H\right]^{2} / \sigma_{i}^{2},
$$

where $\sigma_{i}$ is the error in $\log \left(z_{i} / d_{i}\right)$. The best-fit Hubble constant and the corresponding variance are then

$$
\log H=\sigma^{2} \sum \sigma_{i}^{-2} \log \left(z_{i} / d_{i}\right), \quad \sigma^{2}=\left(\sum \sigma_{i}^{-2}\right)^{-1} .
$$

The scatter about a pure Hubble flow results from both distance uncertainties and peculiar motions. We assume that the distance error is distributed normally in $\log d$, with a standard deviation $\Delta$, where $\Delta$ is the sum in quadrature of the formal distance error of the given $\mathrm{SN}$ and the additional scatter $\Delta_{\text {add }}=0.05 \mathrm{mag}$ adopted as our "standard" case $\left(\Delta_{i}^{2}=\Delta_{\text {MLCS }}^{2}+\Delta_{\text {add }}^{2}\right)$. For the peculiar velocities we assume an rms value, $\sigma_{f}$ (in the frame of the cosmic microwave background [CMB]), independent of distance. For supernovae in the sample beyond $\sim 3000 \mathrm{~km} \mathrm{~s}^{-1}$, the distance error dominates the peculiar velocity uncertainty. Since only six supernovae are closer, we approximate the total scatter of $\log (z / d)$ as normally distributed, with variance $\sigma_{i}^{2}=\Delta_{i}^{2}+\left(\sigma_{f} / z_{i}\right)^{2}$.

Partly motivated by the low dispersion velocity detected for galaxies in redshift and peculiar velocity surveys (Davis \& Peebles 1983; Willick et al. 1997b; Davis, Miller, \& White 1997), we adopt $\sigma_{f}=200 \mathrm{~km} \mathrm{~s}^{-1}$, and explore other values later. This value for $\sigma_{f}$ provides a $\chi^{2}$ per degree of freedom of $\chi_{\text {dof }}^{2}=0.99$ within the inner $100 h^{-1} \mathrm{Mpc}$, where the data and the formal error estimates are most reliable and where $\sigma_{f}$ makes a difference. The contribution of supernovae in the outer regions makes the overall value somewhat larger, $\chi_{\text {dof }}^{2}=1.9$, which suggests that we may underestimate the errors for a few supernovae at very large distances.

\subsection{One-, Two-, and Three-Zone Models}

We try to fit the data with three alternative simple models and compare the corresponding $\chi^{2}:(1)$ a one-zone no-void model of uniform expansion with Hubble constant $H$; (2) a two-zone void model with a local Hubble constant $H_{1}$ within a sphere of radius $R_{1}$, and a global Hubble constant $H$ outside this sphere; and (3) a three-zone void model with an additional shell between the radii $R_{1}$ and $R_{2}$ within which the Hubble constant is $H_{2}$. These models have 1,3 , and 5 free parameters, respectively.

The model parameters are determined by minimizing $\chi^{2}$ (eq. [2]). To maintain at least six supernovae in each zone, $R_{1}$ and $R_{2}$ are limited by the current data to the range 30-200 $h^{-1} \mathrm{Mpc}$. The best-fit models, and the corresponding errors, are plotted in Figure 1. For the two-zone model, the best-fit outflow zone extends out to $R_{1}=70 h^{-1} \mathrm{Mpc}$, and the peculiar monopole in it is $\delta_{H}=0.065 \pm 0.022$, where the error here includes in quadrature the uncertainties in $H_{1}$ and $H$. If SN 1992 bl is excluded, the outflow is somewhat stronger, $\delta_{H}=0.075 \pm 0.022$. The location of $R_{1}$ is extremely robust. When allowing for a third, intermediate zone, the boundary of the inner zone remains robust at $R_{1}=70 h^{-1} \mathrm{Mpc}$, with $\delta_{H}=0.053 \pm 0.022$, and it is surrounded by an inflowing shell of radius $70-105 h^{-1} \mathrm{Mpc}$, within which the inflow amounts to $\delta_{H}=-0.059 \pm 0.027$. (If SN 1992bl is excluded, the extent of the outflow zone would be the same, with $\delta_{H}=0.060 \pm 0.023$, and the inflow shell would be wider, 70-120 $h^{-1} \mathrm{Mpc}$, and somewhat shallower, $\delta_{H}=-0.048 \pm 0.025$.)
The values of $\chi^{2}$ that correspond to these model fits suggest that the monopole deviation from a pure Hubble flow is significant. We obtain $\chi^{2}=80.9,71.5,67.1$ for the three models, respectively. This means a reduction of 4.7 for each additional parameter going from the one-zone to the two-zone model, and an additional reduction of 2.2 per parameter when moving from the two-zone to the threezone model. A reduction in $\chi^{2}$ of more than unity per parameter is commonly regarded as a significant improvement. Yet, because we had a chance to inspect the data before we developed our model, one may argue that only a larger reduction should be considered significant. In addition, the fact that the $\chi_{\text {dof }}^{2}$ at very large distances is somewhat larger than unity complicates the interpretation of the $\chi^{2}$ statistics. In any case, the detected decrease in $\chi^{2}$ indicates that the two-zone model is a significant improvement over the pure Hubble flow model and should be taken seriously, while the three-zone model provides only marginal additional improvement and stretches beyond the statistical reach of the current data set.

If SN $1992 \mathrm{bl}$ were excluded, the corresponding $\chi^{2}$ values would be $67.5,55.2,51.5$, respectively. The improvement associated with the two-zone model would become even more pronounced, with the $\chi^{2}$ being reduced by 6.1 for each additional parameter. The improvement for the three-zone model is still marginal, of 1.9 per additional parameter. The exclusion of SN 1992bl by itself would lead to significantly lower $\chi^{2}$ values, by 13.4 in the one-zone model and by 16.3 in the two-zone model. A jackknife test of excluding one SN at a time and redoing the fits confirms that SN 1992bl is indeed the most deviant data point. However, it does not represent an "abnormal" deviation from a normal distribution.

\subsection{The Significance of a Void within $70 h^{-1} \mathrm{Mpc}$}

Determining the significance of the peculiar monopole implied by the model fits above is not trivial, and we therefore address it in several different ways. One way to assign a more concrete significance is by fixing the radius of interest a priori at $R_{1}=70 h^{-1} \mathrm{Mpc}$, based on the external indications for a local void encompassed by the great walls $(\S 1)$, or on the robustness of $R_{1}$ in the fits to the supernova data themselves.

The statistical question that we pose in this case is, at what confidence level can one reject the null hypothesis that chance alone could produce a fluctuation as large as we see inside $R_{1}=70 h^{-1} \mathrm{Mpc}$, while the underlying field is a pure Hubble flow. A crude answer can be provided by the monopole deviation within $R_{1}$ in units of the corresponding error, the "voidness" $\Phi \equiv \delta_{H} / \sigma$. We obtain $\Phi=3.0$ and 3.5 with and without SN $1992 \mathrm{bl}$ respectively. Thus, for $R_{1}=70 \mathrm{~h}^{-1}$ Mpc fixed by assumption, the $6 \%-7 \%$ outflow is significant at the $3 \sigma$ level.

The assumed value for the velocity dispersion in the inner void region could be decreased once the data have been fitted for a local monopole. The global value of $\sigma_{f}$ was intended to include the contribution from the monopole deviation, which has now been explicitly removed from the velocity error budget within the local void. With a reduced value of $\sigma_{f}=135 \mathrm{~km} \mathrm{~s}^{-1}$ that yields $\chi_{\text {dof }}^{2}=1$ in the inner zone (based on scatter about the best-fit outflow model), the outflow within $70 h^{-1} \mathrm{Mpc}$ becomes even more significant: $\Phi=3.4$ and 3.9 with and without SN $1992 \mathrm{bl}$ respectively. This may be a better estimate of the errors about the best-fit 
model, but to keep our analysis conservative, we do not employ these reduced errors in the estimates of significance that follow.

\subsection{Significance by Gaussian Monte Carlo Analysis}

An alternative, more general approach would be to let the size of the void vary in a given range, then determine the extent of the most significant void from the supernova data themselves, and estimate the probability of observing a void of such depth (of any size in that range). The void size could be determined by minimizing the $\chi^{2}$ of a model that includes $R_{1}$ as a free parameter. Another way to determine the extent and depth of the fluctuation in the monopole (in the two-zone model) is to search for the maximum of $\Phi(R) \equiv \delta_{H}(R) / \sigma(R)$ over $R$ in a search range $\left[r_{\min }, r_{\mathrm{max}}\right] ; \Phi$ is now defined as the maximum value of $\Phi(R)$, and $R_{1}$ is the corresponding $R$. Figure 2 shows $\delta_{H}(R)$ with its error bars (top panel) and the resulting $\Phi(R)$ (bottom panel), both with and without SN 1992bl. The most pronounced monopole perturbation found in this way corresponds to an outflow region of radius $R_{1}=70 h^{-1} \mathrm{Mpc}$.

There is apparently another feature at $R \sim 150 h^{-1} \mathrm{Mpc}$, whose relative significance decreases when SN 1992bl is excluded. This could be an artifact from noisy determination of the universal Hubble constant, since only 13 supernovae lie outside this volume. When determining $H$ instead from all the data points, the value of $\Phi(R)$ at $R \sim 150 h^{-1} \mathrm{Mpc}$ drops to about $60 \%$ of its value at $70 h^{-1}$ Mpc.

When the void size is allowed to be free, the value of $\Phi$ cannot be interpreted simply as the significance of the void, because the radius $R$ has been chosen to maximize $\Phi$.

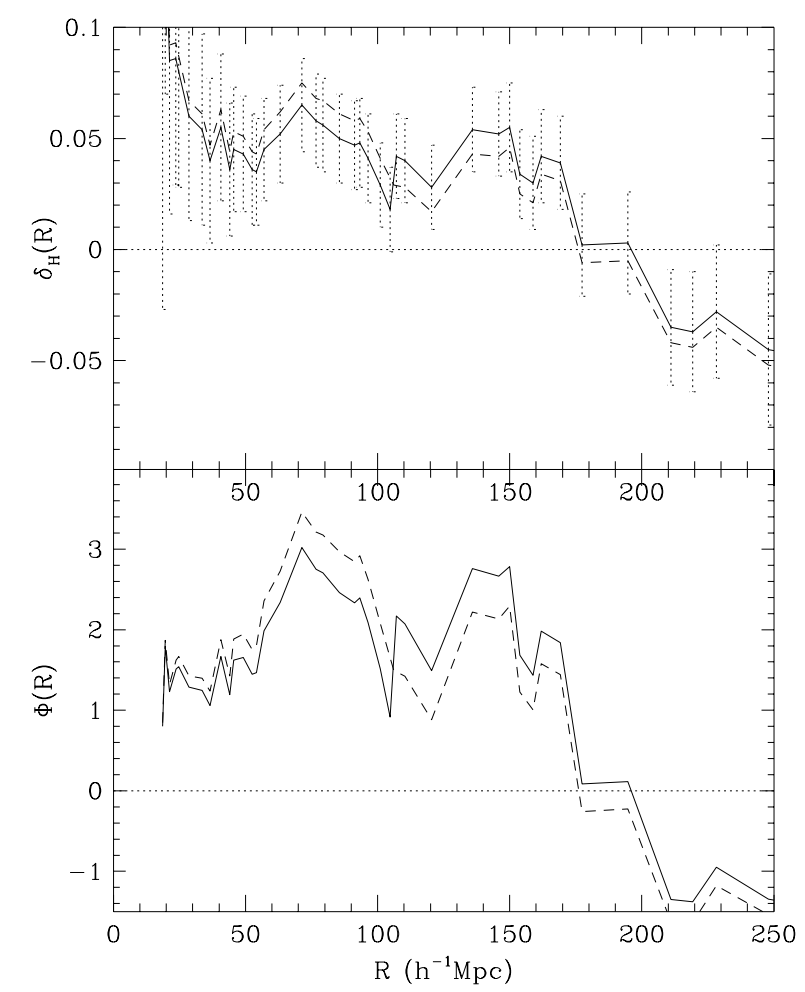

FIG. 2.-Top panel: $\delta_{H}(R)$ vs. radius $R$, for all 44 supernovae (solid line) with the corresponding error bars, and when excluding the outlier (dashed line). $H$ is determined externally to $R$. Bottom panel: $\Phi(R)$ vs. radius $R$, for all 44 supernovae (solid line) and without the outlier (dashed line).
Instead, we employ a Monte Carlo analysis. We generate random synthetic data sets with a single zone of uniform expansion and with the same noise as in our real data. We then compute the statistic $\Phi$ for each realization while allowing $R$ to vary in a given search range, construct the probability distribution function of $\Phi$, and see how far in the tail of this distribution the observed maximum $\Phi$ lies. The corresponding percentile, $p$, is the probability that the void is a chance occurrence. (This probability refers only to outflows of voidness greater than $\Phi$, not including the opposite tail of the distribution of inflows, below $-\Phi$.) The maximum search range allowed by the data is roughly 30-200 $h^{-1}$ Mpc, which imposes only a weak initial constraint on $R$. We may also choose to try a sequence of more limited search ranges, all the way to a tight range about $R=70 h^{-1} \mathrm{Mpc}$, which would bring us back to the alternative approach of focusing attention on a predetermined scale.

Ten thousand Monte Carlo realizations of the data are created by perturbing a pure Hubble flow with a Gaussian distribution of errors. The synthetic supernovae are initially placed at the redshift-space positions of the 44 observed supernovae. The distance moduli are then perturbed by a Gaussian random variable of standard deviation equal to the assumed distance error of that supernova, and $z$ is perturbed further by a Gaussian of width $\sigma_{f}$ to account for peculiar velocities. The Monte Carlo realizations aim at reproducing the same biases that arise in the analysis of the real data due to coupling between the distance errors and the deviations from Hubble flow.

For the most general search, allowing $R$ to be anywhere in the range $30-200 h^{-1} \mathrm{Mpc}$, we find $p=0.022$, that is, there is a $2.2 \%$ likelihood that the void is a chance occurrence. (When SN 1992bl is excluded, this probability reduces to $p=0.5 \%$.) When the search is limited to the intermediate range $50-100 h^{-1} \mathrm{Mpc}$, the probability becomes $0.9 \%$ (and $0.2 \%$ without SN $1992 \mathrm{bl}$ ).

The Gaussian synthetic catalogs are straightforward analogs of the real data, but their validity relies on the accuracy of our error estimates and their distribution. The fact that the observed $\chi^{2}$ per degree of freedom is of order unity for the pure Hubble flow model and our assumed errors, indicates that our assumed errors are not unrealistic. The probability distribution of the observed $\log (z / d)$ is nearly indistinguishable from a normal distribution, except, perhaps, for the marginal outlier SN 1992bl. This suggests that our synthetic catalogs properly represent the noisy data and that the corresponding significance estimate is a useful guide. In $\S 4$ we briefly explore the effect of using smaller or larger errors.

\subsection{Error Estimation by Bootstrap Resampling}

Another way to evaluate significance that does not rely so heavily on an assumed knowledge of the errors is to estimate the standard deviation in the statistic $\Phi$ by bootstrap resampling of the noisy data themselves, and using that estimate to evaluate the deviation of the observed $\Phi$ from the null hypothesis of $\Phi=0$. (We do not use the bootstrap realizations to derive the whole probability distribution, the way we did with the Gaussian realizations, because it could be biased.)

We create 10,000 synthetic catalogs by randomly selecting 44 data points from the real data, allowing for repetitions. We then calculate the $\Phi$ statistic for each realization, 
and adopt the standard deviation over the realizations as $\sigma_{\Phi}$

We note that this bootstrap analysis is not completely independent of the assumed errors, as they enter explicitly in the definition of $\Phi(R)$ and in the $\chi^{2}$ fitting. However, because the realizations are based on the real noise, this provides a partly independent way to test the significance of the void.

The error estimated this way is $\sigma_{\Phi}=1.2$. It is slightly larger than unity because $R$ is chosen in each realization to maximize $\Phi$. This value of $\sigma_{\Phi}$ implies that the confidence level by which the observed $\Phi=3.0$ is different from $\Phi=0$ is $\sim 2.5 \sigma$, or that the probability of obtaining such a void by chance is roughly $0.6 \%$. (If SN $1992 \mathrm{bl}$ is excluded, the result is $\Phi=3.5 \pm 1.1$, which can be similarly interpreted as a 3.1 $\sigma$ effect, of $0.1 \%$ probability.)

The bootstrap realizations show good evidence for significant voids at values of $R$ near $70 h^{-1} \mathrm{Mpc}$, as well as near $150 h^{-1} \mathrm{Mpc}$ (see Fig. 2), because each of these regions contains many data points that indicate a real effect. The void significance based on the bootstrap analysis is therefore similar to the significance found from the Gaussian synthetic catalogs when the search range of $R$ is narrow about the characteristic scale of the observed data, and higher than the significance found when the search in $R$ is wide.

The bootstrap analysis can also help us evaluate the robustness of the void size. Since the $R$ of maximum $\Phi$ in the bootstrap realizations sometimes occurs near $150 h^{-1}$ $\mathrm{Mpc}$ rather than near $70 h^{-1} \mathrm{Mpc}$, the error in the radius of the $70 h^{-1} \mathrm{Mpc}$ void is hard to estimate when the search range includes the two characteristic scales. When the 150 $h^{-1} \mathrm{Mpc}$ vicinity is excluded from the search range, the bootstrap distribution yields $R=70 \pm 7 h^{-1} \mathrm{Mpc}$. The uncertainty is set by the coarseness of our data sampling and is roughly equal to the distance between the two supernovae closest to $70 h^{-1} \mathrm{Mpc}$.

\section{CAUTIONS}

\subsection{Other Cases}

Table 1 summarizes the results of applying the tests described in $\S 3$ to several other cases where we vary the cosmological parameters, the search range of void extent, the inclusion of the outlier SN 1992bl, and the assumed distance errors. The cosmological density parameters $\Omega_{m}$ and $\Omega_{\Lambda}$ enter via the "luminosity" distance (eq. [1]), which is approximately a function of the deceleration parameter, $q_{0}=\Omega_{m} / 2-\Omega_{\Lambda}$. The outlier SN $1992 \mathrm{bl}$ is alternately included or excluded. We test two options for the search range of $R$ : wide $(30,200)$, and narrow $(60,80)$. The input errors vary between the formal errors of the MLCS method $\left(\Delta_{\mathrm{add}}=0\right)$ and a generous overestimate of the errors, with $\Delta_{\text {add }}=0.1 \mathrm{mag}$. The corresponding values of $\sigma_{f}=250$ and $120 \mathrm{~km} \mathrm{~s}^{-1}$, respectively, were obtained such that $\chi_{\text {dof }}^{2} \sim 1$ within the inner region (compared to a pure Hubble flow).

Listed are the resulting values of $\delta_{H}$ and $\Phi$ corresponding to the best-fit inner zone (independent of the search range), the error in $\Phi$ based on the bootstrap resampling, $\sigma_{\Phi}$, and the (one-sided) probability $p$ that the void is a chance occurrence based on the Gaussian Monte Carlo realizations.

The derived size of the void is insensitive to the choice of parameters in Table 1 . The void in all cases encompasses the first 17 supernovae and is of radius $R \simeq 70 h^{-1} \mathrm{Mpc}$. The global Hubble constant $H$, determined externally to the void, is robust at $\sim 65 \pm 1 \mathrm{~km} \mathrm{~s}^{-1} \mathrm{Mpc}^{-1}$. (The error refers only to the $\chi^{2}$ fit, and the sensitivity to the parameters that vary in Table 1 is even smaller. This value of the Hubble constant is based on the original calibration used by RPK, before Hipparcos.) Constraining the search range for $R$ has the effect of increasing the void significance based on the Gaussian Monte Carlo realizations, e.g., from $p=2.2 \%$ to $p=0.5 \%$ for our standard case. The standard deviation $\sigma_{\Phi}$ as estimated by the bootstrap resampling is less sensitive to the search range. The exclusion of the outlier SN 1992bl increases the significance of the void in all cases.

Although most of the supernovae are within $z<0.1$, the cosmological effects are important because we are dealing with a small fluctuation of a few percent. Making no cosmological correction is equivalent to assuming $\Omega_{m}=2$ and $\Omega_{\Lambda}=0$. The depth and significance of the void both decrease gradually for smaller $q_{0}$. The peculiar monopole $\delta_{H}$ goes down from $7.5 \%$ (with no cosmological correction) to $5 \%$ (for $\Omega_{m}=\Omega_{\Lambda}=0.5$ ). In the case $\Omega_{m}=\Omega_{\Lambda}=0.5$, as long as we keep the outlier in and perform a wide search for $R$, the void becomes only marginally significant, with $p$ as high as $\sim 9.8 \%$. However, even in this case, the void

TABLE 1

Two-Zone Model Results for Various Cases

\begin{tabular}{|c|c|c|c|c|c|c|c|c|}
\hline$\Delta_{\text {add }}$ & $\Omega_{m}$ & $\Omega_{\Lambda}$ & SN 1992bl & $r_{\min }-r_{\max }$ & $\underset{(\%)}{\delta_{H} \pm \sigma}$ & $\Phi$ & $\pm \sigma_{\Phi}$ & $\begin{array}{c}\text { Gaussian } p \\
(\%)\end{array}$ \\
\hline \multirow[t]{3}{*}{$0.05 \ldots \ldots$} & \multirow[t]{3}{*}{1} & \multirow[t]{3}{*}{0} & \multirow[t]{2}{*}{ In } & $30-200$ & $6.5 \pm 2.2$ & 3.0 & 1.2 & 2.2 \\
\hline & & & & $\begin{array}{l}60-80 \\
30-200\end{array}$ & $75-$ & 3.5 & $\begin{array}{l}1.2 \\
1.1\end{array}$ & $\begin{array}{l}0.5 \\
0.5\end{array}$ \\
\hline & & & Out & $60-80$ & & & 1.2 & 0.1 \\
\hline \multirow[t]{4}{*}{$0.05 \ldots \ldots$} & \multirow[t]{4}{*}{0.4} & \multirow[t]{4}{*}{0} & \multirow[t]{2}{*}{ In } & $30-200$ & $5.9 \pm 2.1$ & 2.8 & 1.2 & 4.3 \\
\hline & & & & $60-80$ & & & 1.2 & 1.2 \\
\hline & & & \multirow[t]{2}{*}{ Out } & $30-200$ & $6.9 \pm 2.2$ & 3.2 & 1.2 & 1.2 \\
\hline & & & & $60-80$ & & & 1.2 & 0.3 \\
\hline \multirow[t]{4}{*}{$0.05 \ldots \ldots$} & \multirow[t]{4}{*}{0.5} & \multirow[t]{4}{*}{0.5} & \multirow[t]{2}{*}{ In } & $30-200$ & $5.1 \pm 2.1$ & 2.4 & 1.2 & 9.8 \\
\hline & & & & $60-80$ & & & 1.2 & 3.0 \\
\hline & & & \multirow[t]{2}{*}{ Out } & $30-200$ & $6.1 \pm 2.2$ & 2.8 & 1.1 & 3.5 \\
\hline & & & & $60-80$ & & & 1.2 & 0.9 \\
\hline \multirow[t]{2}{*}{$0.05 \ldots \ldots$} & \multirow[t]{2}{*}{2} & \multirow[t]{2}{*}{0} & In & $30-200$ & $7.5 \pm 2.2$ & 3.5 & 1.2 & 0.6 \\
\hline & & & Out & $30-200$ & $8.5 \pm 2.2$ & 3.9 & 1.1 & 0.1 \\
\hline $0 \ldots \ldots \ldots$ & 1 & 0 & In & $30-200$ & $6.6 \pm 2.2$ & 3.0 & 1.4 & 1.8 \\
\hline $0.1 \ldots \ldots$ & 1 & 0 & In & $30-200$ & $6.3 \pm 2.3$ & 2.7 & 1.0 & 6.5 \\
\hline
\end{tabular}


becomes more significant, with $p=3 \%-3.5 \%$, if one either excludes the outlier or limits the search range.

If one tries the formal errors only, the significance based on the Gaussian synthetic catalogs becomes slightly larger than in the standard case studied above; $p=1.8 \%$ for $\Delta_{\text {add }}=0$ compared to $2.2 \%$ for $\Delta_{\text {add }}=0.05$. Increasing $\Delta_{\text {add }}$ to 0.1 makes the significance weaker, $p=6.4 \%$. The significance is increased back to the $p \sim 2 \%$ level by either excluding the outlier or limiting the search range. The response of evaluation based on bootstrap to the assumed errors is more complicated because these errors enter $\Phi$ and $\chi^{2}$. The value of $\Phi / \sigma_{\Phi}$ can be crudely translated to a one-sided probability for a chance occurrence by assuming a Gaussian distribution, yielding $p=1.8 \%$ with $\Delta_{\text {add }}=0$ and $p=0.3 \%$ for $\Delta_{\text {add }}=0.1$. The fact that the bootstrap evaluation for $\Delta_{\text {add }}=0.1$ indicates a considerably higher significance than obtained from the Gaussian synthetic catalogs suggests that the errors assumed in the Gaussian model are too large.

\subsection{Higher Multipoles}

One concern in the interpretation of our result is that the sparse sampling and the incomplete sky coverage (especially at low Galactic latitudes) may introduce a bias in the peculiar monopole due to its covariance with higher multipoles (e.g., Feldman \& Watkins 1994, 1998). Figure 3 is an Aitoff plot of the angular positions of the 27 inner supernovae, in the void and in the shell around it, with their peculiar velocities indicated.

The fact that a similar monopole signal is seen in both the north and the south Galactic caps suggests that the mixup with a bulk flow is not likely to be severe. To quantify the sensitivity of the monopole to a possible bulk velocity of a similar extent, we redo the two-zone model fit with an additional free vector of bulk velocity (in the CMB frame) within each zone. We find that $\delta_{H}$ within $70 h^{-1} \mathrm{Mpc}$ typically becomes lower by only about $1 \%$, and the void significance is reduced.

While the monopole can be properly determined out to large distances, the bulk velocity is much harder to determine at large distances. This is because the relevant residuals for the monopole are the errors in $\delta_{H}$, which are largely independent of distance, whereas the residuals relevant for the bulk velocity are the errors in the absolute peculiar velocities, which are generally proportional to distance. The best-fit bulk velocity in the void is found to be $(200 \pm 300) \mathrm{km} \mathrm{s}^{-1}$ (note the large error!), in the general direction of the Local Group motion relative to the CMB frame and in agreement with a straightforward extrapolation of the bulk velocity found from galaxy peculiar velocities in the inner $60 h^{-1} \mathrm{Mpc}$ (Dekel et al. 1998), but not much different from zero.

We have also checked the effect of subtracting out from the data a bulk flow that is fixed by assumption, based on the extrapolation of the galaxy bulk flow from the inner 60 $h^{-1} \mathrm{Mpc}$, namely, $200 \mathrm{~km} \mathrm{~s}^{-1}$ in the direction of the CMB dipole. Subtracting such a bulk velocity from the supernova velocities inside the void produces an effect on the void depth that is similar to the case when the bulk velocity was free in the fit. The probability of the outflow monopole being a chance occurrence is increased from $p=2.2 \%$ to about $p=7 \%$ in this case.

To investigate further the possible cross-talk with bulk velocity, we generate synthetic data from a toy model whose "true" velocity field is the same as our best-fit two-zone model, namely, a pure Hubble flow outside $70 h^{-1} \mathrm{Mpc}$ and a $6.5 \%$ faster Hubble flow inside. We alternatively add to the "true" Hubble flow model a constant bulk velocity. This toy flow model is "observed," with the appropriate errors, at the real positions of the 44 supernovae, and our statistical analysis is applied. The effect of the bulk flow on the solution for the monopole is found to be weak. When no bulk flow is present in the "true" toy model and no bulk velocity is allowed in the model fit, we find that the void is reproduced properly. Then, when allowing for a free bulk velocity in the fit, the correct void is reproduced, independent of whether a bulk velocity was present in the "true" toy model. However, when an assumed bulk velocity is subtracted out from the toy data and then a monopole is fitted, the void is reproduced accurately only when the correct bulk flow is assumed. For example, when the procedure is

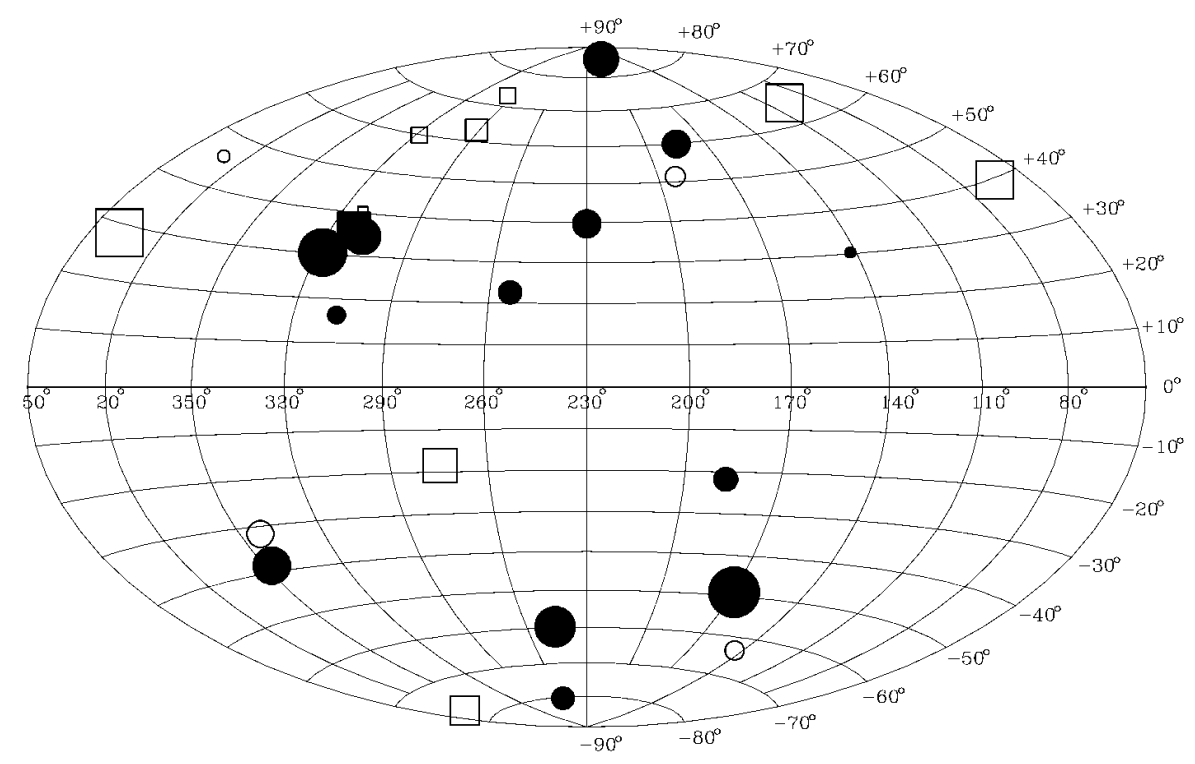

FIG. 3.-Angular positions of the supernovae, in Galactic coordinates, inside the void $d<70 h^{-1} \mathrm{Mpc}$ (circles) and in the shell around it, $70<d<120$ $h^{-1} \mathrm{Mpc}$ (squares). Filled and open symbols mark outward and inward peculiar velocities, respectively, and the size of the symbol is proportional to the absolute value of the peculiar velocity in the CMB frame. 
applied to a toy model of no "true" bulk flow and a $200 \mathrm{~km}$ $\mathrm{s}^{-1}$ bulk velocity is assumed in the fit, the result underestimates the depth of the void by $\sim 1 \%$. When there is a "true" bulk flow in the model which is not allowed in the fit, the depth is overestimated by $\sim 1 \%$. These tests confirm our expectation that, although some covariance between monopole and dipole is inevitable given the specific sampling positions of these 44 supernovae, the effect on the recovered void is small.

The possible confusion with a quadrupole moment is worse. The poor coverage at low and moderate Galactic latitudes (e.g., at $0^{\circ}<l<170^{\circ}$ and $-60^{\circ}<b<+30^{\circ}$ which includes the Perseus-Pisces region) makes it practically impossible to distinguish between a peculiar monopole and a quadrupole. Future observations which improve the coverage of regions of moderate Galactic latitudes would help constrain the quadrupole. We note that previous investigations of the quadrupole flow (e.g., Lilje, Yahil, \& Jones 1986) have pointed to a pattern that is almost orthogonal to an outflow at the Galactic poles that would be mistaken for our monopole. We also know that the mass distribution in the Galactic plane is dominated by two big attractors in opposite sides of the sky, the Great Attractor and the Perseus-Pisces supercluster, which would rather induce an inflow along the Galactic poles. The current data set is too small for a quantitative analysis of possible aliasing by quadrupole and higher multipoles, which is left for future analysis with more data.

\section{DISCUSSION}

We have analyzed the monopole perturbations in the peculiar velocity field as probed by $44 \mathrm{SNe}$ Ia with small distance errors. For an Einstein-de Sitter universe, we find an outward perturbation in the Hubble flow of $(6.5 \pm 2.2) \%$ within a sphere of radius $70 \mathrm{~h}^{-1} \mathrm{Mpc}$, with a possible indication for inflow in the surrounding shell. The void is less significant if $\Omega_{m}$ is low and $\Omega_{\Lambda}$ is high. Its significance is increased when one outlier is excluded, or when the size of the void is constrained a priori to a limited range.

In the idealized case of a spherically symmetric density distribution about the Local Group, an observed monopole in the velocity field is associated with a specific density profile. In linear theory, $\delta \rho / \rho=-\nabla \cdot v / \Omega^{0.6}$, so that a constant velocity monopole $\delta_{H}$ corresponds, for $\Omega=1$, to a constant density fluctuation offset of $\delta \rho / \rho=-3 \delta_{H}$. In this picture, our results would require a local void of $\sim-20 \%$ density contrast.

In general, when the actual density distribution is not spherisymmetric about the observer, there is no general deterministic relation between the peculiar Hubble flow and the density profile within a given volume, and one should appeal to a statistical relation between the two. Shi, Widrow, \& Dursi (1996) find, in the framework of linear theory, and for typical cosmological models with $\Omega=1$, that on average about a random point in space, the relation is $\delta_{H} \approx 1.7 \delta \rho / \rho$, i.e., with the factor 3 replaced by $\approx 1.7$. A similar result has been obtained from simulations by Turner et al. (1992). Thus, if the Local Group is assumed to be a random point in space that is not necessarily at the center of a void, our observed $\delta_{H} \sim 6.5 \%$ within a sphere of radius $70 h^{-1} \mathrm{Mpc}$ would be translated, on average, to a local underdensity of only $-11 \%$ (for $\Omega=1$ ). A lower $\Omega$ would lead in both cases to a deeper underdensity by a factor of about $\Omega^{-0.6}$.
One could argue that the data provide some evidence for our being near the center of a roughly spherical void. As discussed above, the outflow detected in both the north and the south is indicative of a spherical outflow, with a limited dipole signature. Furthermore, there is no indication in Figure 1 for significant radial deviations from a constant $\delta_{H}$ within the volume of the void. Finally, the Great Walls do appear in redshift space to define a crude spherical shell of galaxies centered roughly about the Local Group. This preliminary evidence that we are near the "center" of a roughly spherical void makes the estimate of $\sim-20 \%$ more likely. Because of the small number of data points in the current sample, we have not attempted to determine the center point about which the angle-averaged void is deepest or largest.

The sharp drop in $\delta_{H}$ at $R=70-75 h^{-1}$ Mpc would be produced by a narrow shell of high mass density at that distance. This is approximately the observed location of the shell-like structure defined in redshift surveys by the Great Wall of Coma and the Southern Wall. It is tempting to associate the drop in the outflow we see with these features of the regional galaxy distribution. Indeed, Dell'antonio, Geller, \& Huchra (1996), based on a sample of peculiar velocities of spiral galaxies in the vicinity of the Great Wall, estimate the real-space width of the wall to be smaller than $11 h^{-1} \mathrm{Mpc}$ at $90 \%$ confidence. Their corresponding limit on the overdensity of the Great Wall is $\delta \rho / \rho<2 / \Omega^{0.6}$.

An outer region of negative mean $\delta_{H}$ beyond $75 h^{-1} \mathrm{Mpc}$ would represent back-streaming from voids behind the walls into the walls. We expect that the density profile averaged over a sphere returns to its mean value $(\delta M / M=0)$ where the velocity monopole converges to the global Hubble flow.

The evidence for such a local void in density profiles derived from redshift surveys is not conclusive. While we see no evidence for a local void in the IRAS 1.2 Jy survey as analyzed by Koranyi \& Strauss (1997, Fig. 6), Springel \& White (1997, Fig. 5) clearly show evidence for a void based on the same data. A similar void is seen in Saunders et al. (1990, Fig. 11) based on the QDOT-IRAS redshift survey. Preliminary results from the PCSz-IRAS redshift survey provide further evidence for the presence of a local void of even larger extent (S. D. M. White 1997, private communication). The apparently conflicting results could be partly explained by the fact that the effective survey size, especially in the $1.2 \mathrm{Jy}$ survey, is not much larger then the volume of the local void. This might introduce some confusion between the radial selection function and the real density profile.

Such a void is not in significant conflict with expectations from standard cosmological theories. The expected rms perturbations on these scales, as predicted by popular cold dark matter (CDM) models, range from $2 \%$ to $4 \%$, with standard CDM $\left(\Omega_{m}=1\right)$ being on the high side and open $\operatorname{CDM}\left(\Omega_{m}=0.3, \Omega_{\Lambda}=0\right)$ on the low side (see also Shi \& Turner 1998; Wang, Spergel, \& Turner, 1998). The local void we find, of $\sim 6 \% \pm 2 \%$, is thus less than a $2 \sigma$ deviation from the predictions of any of the conventional models. Although an open model is less likely than the others, the void in this case is slightly shallower and corresponds only to a $\sim 1.9 \sigma$ deviation from the predictions of an open CDM model.

One should also worry about possible observational systematics that may be artificially interpreted as a local pecu- 
liar outflow. Sources of observational error that vary systematically with distance are prime suspects. These include the effects of redshift on the integrated spectral light which passes through a fixed broadband filter, the " $K$ correction." Although care has been taken to include such corrections, the nonstellar features of a SN Ia spectrum inhibit an exact compensation for this effect. However, the magnitude of the correction is fairly small for the bulk of our supernova data, typically less than 0.02 mag (Hamuy et al. 1993b). This corresponds to a peculiar velocity of $\sim 50$ $\mathrm{km} \mathrm{s}^{-1}$, which is less than $1 \%$ of deviation from Hubble flow within the local void compared to the detected signal of $\sim 6 \%$ deviation. Our estimate of $10 \%$ error in the $K$ correction implies that these effects are negligible.

Similarly, the range of supernova luminosities observed in the nearby sample is larger than at great distances because it includes intrinsically faint objects. Any defects in the ability of MLCS to correctly account for these systematic shifts could, in principle, cause problems in making reliable inferences about the void dynamics. However, this does not seem to be an important effect. The faint tail in the inner region is populated by only three supernovae, of which only one, the brightest of the three, has a significant positive $\delta H / H$.

We have also tested for correlations between the distances or peculiar velocities and certain quantities that might have systematically affected them. These quantities include the supernova detection time with respect to the time of the light-curve maximum, the absolute correction to the light-curve template, and the magnitude of the extinction correction. We have found no significant correlations.

One implication of our result is that the local value of the Hubble constant cannot be much larger than the global value. Our best estimate of $\delta_{H}$ is $(6.5 \pm 2.2) \%$, so if the regional value (inside $70 \mathrm{~h}^{-1} \mathrm{Mpc}$ ) were $70 \mathrm{~km} \mathrm{~s}^{-1} \mathrm{Mpc}^{-1}$, the global value would be $65 \mathrm{~km} \mathrm{~s}^{-1} \mathrm{Mpc}^{-1}$. But for the global value to reach $50 \mathrm{~km} \mathrm{~s}^{-1} \mathrm{Mpc}^{-1}$ would require a $\delta_{H}$ of $40 \%$, which is clearly excluded by our analysis. This assumes that the volume sampled by our set of SNe Ia reaches out to the mean density and that we are not embedded in a deeper void of even larger extent. A possible hint for such a void may come from the observed overabundance in faint galaxy counts, which may, or may not, be explained by luminosity evolution of galaxies (e.g., Heydon-Dumbelton, Collins, \& McGillivray 1989; Maddox et al. 1990; Lilly 1993; Driver, Windhorst, \& Griffiths 1995; Gronwall \& Koo 1995). One controversial interpretation is of a significant deficiency of galaxies on size scales of $\sim 300 h^{-1} \mathrm{Mpc}$ (Huang et al. 1997). However, in the Las Campanas Redshift Survey, the galaxy density profile clearly converges to a universal value beyond 150 $h^{-1} \mathrm{Mpc}$ (Lin et al. 1996, Fig. 8). Furthermore, a comparison of supernova distances at low and high redshifts puts an upper limit of $10 \%$ at $95 \%$ confidence on the depth of a void of size $\sim 300 h^{-1} \mathrm{Mpc}$ (Kim et al. 1997). Our tentative local void detection is also consistent with upper limits on $\delta_{H}$ of $\sim 7 \%$ obtained from Abell/ACO clusters (Lauer \& Postman 1992; Lauer et al. 1998).

The $\sim 6.5 \%$ outflow detected here would partially help reconcile the large-scale estimates of the Hubble constant, e.g., from supernovae (Tammann \& Sandage 1995; Riess et al. 1996) and estimates that are limited more to the inner volume, such as estimates based on Tully-Fisher distances (Kennicutt et al. 1995; Mould et al. 1995; Freedman et al. 1994; Freedman 1997; Giovanelli et al. 1998). The latter are typically $\sim 10 \%-20 \%$ higher. The local void marginally detected here would help, in a humble way, in the resolution of the apparent conflict between the Hubble constant and the ages of globular clusters (Bolte \& Hogan 1995; Chaboyer et al. 1998). The implication for the value of $\Omega$ is that estimates based on data within the local void (see a review by Dekel, Burstein, \& White 1997) would underestimate the universal value by $\sim 20 \%$.

We conclude by reiterating that our detection of the local void is only marginal. Since its statistical significance can be interpreted in several different ways, we presented a "supermarket" of statistical evaluations. If we were investigating the properties of a void whose scale had been established by other data, such as the great walls seen in redshift slices and pencil beams, we would report a $\sim 6.5 \%$ outflow, which, in an Einstein-de Sitter universe, has a probability of order $1 \%$ to be obtained by chance from a pure Hubble flow. The void would be even more significant if one outlier were removed. However, with no preconception about the extent of the void, the detection is less decisive, with the probability for a chance occurrence rising to a few percent. For the low $\Omega_{m}$ and high $\Omega_{\Lambda}$ models this probability is as high as $10 \%$, and in every case there is possible confusion with higher moments of the velocity field, especially a quadrupole. In any case, our indication of a modest local void is tentative and should be confirmed (or refuted) by future observations; our detection poses a specific model to be tested, that of an outflowing region of radius $70 h^{-1} \mathrm{Mpc}$.

We thank George Blumenthal, Bill Press, and Xiangdong Shi for stimulating discussions. This research was supported in part by the US-Israel Binational Science Foundation grant 95-00330, by the Israel Science Foundation grant 950/95, by a NASA Theory grant at UCSC, by NSF grants AST 95-28899 and AST 96-17058 at Harvard University, and by NSF grant PHY 94-07194 at the Institute for Theoretical Physics, Santa Barbara.
Bartlett, J. G., Blanchard, A., Silk, J., \& Turner, M. S. 1995, Science, 267, 980

Bolte, M., \& Hogan, C. J. 1995, Nature, 376, 399

Branch, D., Nugent, P., \& Fisher, A. 1997, in Thermonuclear Supernovae, ed. R. Canal, P. Ruiz-Lapuente, \& J. Isern (Dordrecht: Kluwer), 715

Broadhurst, T. J., Ellis, R. S., Koo, D. C., \& Szalay, A. S. 1990, Nature, 343, 726

Chaboyer, B., Demarque, P., Kernan, P. J., \& Krauss, L. M. 1998, ApJ, 494, 96

da Costa, L. N., et al. 1994, ApJ, 424, L1

Davis, M., Miller, A., \& White S. D. M. 1997, ApJ, 490, 63

Davis, M., \& Peebles, P. J. E. 1983, ApJ, 267, 465

Dekel, A., Burstein, D., \& White, S. D. M. 1997, in Critical Dialogs in Cosmology, ed. N. Turok (Singapore: World Scientific), 175

Dekel, A., Eldar, A., Kolatt, T., Willick, J. A., Faber, S. M., Corteau, S., \&

Burstein, D. 1998, in preparation

Dell'antonio, I. P., Geller, M. J., \& Bothun G. D. 1996, AJ, 112, 1780

\section{EFERENCES}

Driver, S. P., Windhorst, R. A., \& Griffiths, R. E. 1995, ApJ, 453, 48

Einasto, J., et al. 1997, Nature, 385, 139

Falco, E. E., Shapiro, I. I., Moustakas, L. A., \& Davis, M. 1997, ApJ, 484, 70

Feldman, H. A., \& Watkins, R. 1994, ApJ, 430, L17 1998, ApJ, 494, L129

Freedman, W. L. 1997, in Critical Dialogues in Cosmology, ed. N. Turok (Singapore: World Scientific), 92

Freedman, W. L., et al. 1994, Nature, 371, 757

Geller, M. J., \& Huchra, J. P. 1989, Science, 246, 897

Giovanelli, R., Hayes, M. P., da Costa, L. N., Freudling, W., Salver, J. J., \& Wegner, G. 1997, ApJ, 477, L1

Gronwall, C., \& Koo, D. C. 1995, ApJ, 440, L1

Hamuy, M., et al. 1993a, AJ, 106, 2392

Hamuy, M., Phillips, M. M., Maza, J., Suntzeff, N. B., Schommer, R. A., \& Aviles, A. 1995, AJ, 109, 1

Hamuy, M., et al. 1996a, AJ, 112, 2408 
Hamuy, M., Phillips, M. M., Suntzeff, N. B., Schommer, R. A., Maza, J., \& Aviles, R. 1996b, AJ, 112, 2398

Hamuy, M., Phillips, M. M., Wells, L. A., \& Maza, J. 1993b, PASP, 105, 787

Heydon-Dumbelton, N., Collins, C. A., \& McGillivray, H. 1989, MNRAS, 238,379

Hu, W., \& White, M. 1996, ApJ, 471, 30

Huang, J.-S., Cowie, L. L., Gardner, J. P., Hu, E. M., Songaila, A., \& Wainscoat, J. 1997, ApJ, 476, 12

Huchra, J., Davis, M., Latham, D. W., \& Tonry, J. 1983, ApJS, 52, 89

Jacoby, G. H., et al. 1992, PASP, 104, 678

Keeton C. R., \& Kochanek, C. S. 1997, ApJ, 487, 42

Kennicutt, R. C., Freedman, W. L., \& Mould, J. R. 1995, AJ, 110, 1476

Kim, A. G., et al. 1997, ApJ, 476, L63

Kirshner, R. P., Oemler, A., Schechter, P. L., Shectman, S. A. 1981 ApJ, 248, L52

Koranyi, D. M., \& Strauss, M. A. 1997, ApJ, 477, 36

Landy, S. D., Shectman, S. A., Lin, H., Kirshner, R. P., Oemler, A., \& Tucker, D. 1996, ApJ, 456, L1

Lauer, T. R., \& Postman, M. 1992 ApJ, 400, L47

. 1994, ApJ, 425, 418

Lauer, T. R., Tonry, J. L., Postman, M., Ajhar, E. A., \& Holtzman, J. A. 1998, ApJ, 499, 577

Lilje, P. B., Yahil, A., \& Jones, B. J. T. 1986, ApJ, 307, 91

Lilly, S. J. 1993, ApJ, 411, 501

Lin, H., Kirshner, R. P., Shectman, S. A, Landy, S. D., Oemler, A., Tucker, D. L., \& Schechter, P. L. 1996, ApJ, 464, 60
Maddox, S. J, Sutherland, W. J., Efstathiou, G., Loveday, J., \& Peterson, B. A. 1990, MNRAS, 247, 1P

Mould, J. R., et al. 1995, ApJ, 449, 413

Riess, A. G. 1996, Ph.D. thesis, Harvard Univ

Riess, A. G., Davis, M., Baker, J., \& Kirshner, R. P. 1997, ApJ, 488, L1

Riess, A. G., Press W. H., \& Kirshner, R. P. 1995a, ApJ, 438, L17 .1995b, ApJ, 445, L91 . 1996, ApJ, 473, 88 (RPK)

Riess, A. G., et al. 1998, in preparation

Sandage, A., \& Tammann, G. A. 1993, ApJ, 415, 1

Saunders, W., Rowan-Robinson, M., Lawrence, A., Efstathiou, G., Kaiser, N., Ellis, R. S., \& Frenk, C. S. 1990, MNRAS, 242, 318

Shectman, S. A., Landy, S. D., Oemler, A., Tucker, D. L., Lin, H., Kirshner, R. P., \& Schechter, P. L. 1996, ApJ, 470, 172 S

Shi, X. 1997, ApJ, 486, 32

Shi, X., \& Turner, M. S. 1998, ApJ, 493, 519

Shi, X., Widrow, L. M., \& Dursi, L. J. 1996, MNRAS, 281, 565

Sigad, Y., Eldar, A., Dekel, A., Strauss, M. A., \& Yahil, A. 1998, ApJ, 495, 516

Springel, V., \& White, S. D. M. 1997, MNRAS, submitted (astro-ph/ 9704126)

Tammann, G. A., \& Sandage, A. 1995, ApJ, 452, 16

Turner, E. L., Cen, R., \& Ostriker, J. P. 1992, AJ, 103, 1427

Wang, Y., Spergel, D. N., \& Turner, E. L. 1998, ApJ, 498, 1

Willick, J. A., Courteau, S., Faber, S. M., Burstein, D., Dekel, A., \& Strauss, M. S. 1997a, ApJS, 109, 333

Willick, J. A., Strauss, M. S., Dekel, A., \& Kolatt, T. 1997b, ApJ, 486, 629 